\title{
Contents, Vol. 9, 1952
}

\section{INDEX}

Amsler, M., Augen-Tuberkulose

359

Baroni, V., Experiences cliniques et de laboratoire avec 1'H.I.N

Böhní, E., vide Fust, B.

Bonard, E. C, Discussion se rapportant aux conferences sur le Rimifon . . 295

Brunner, A., Die Lungenresektion bei Tuberkulose

Brunner, W., Uber die Einpflanzung von frischem Fremdspan bei Knochenund Gelenktuberkulose 330

Brunner, W., Die Prinzipien der chirurgischen Behandlung der Knochen- und

Gelenktuberkulose 399

Brunner, W., Lungenresektion bei Tuberkulose

Burckhardt, W., Die Tuberkulose der Haut 434

de Beule, R., Problème de la resistance du bacille tuberculeux aux medica ments en general et au PAS en particulier 76

Decker, P., L'exérèse pulmonaire pour tuberculose 506

Delachaux, A. et P. Jaquíer, Reactions vaccinales et maladies tuberculeuses observées après inoculation BCG 203

Düggelí, 0. und F. Trendelenburg, Vergleichende Frühergebnisse der RimifonBehandlung bei Lungentuberkulose 267

Fehr, A. M., Differentialdiagnose der Kniegelenksaffektionen

Fíerz, F., Primärtuberkulose bei eineiigen Zwillingen

Frank, P., Foyers extra-articulaires des os longs 319

Fust, B., A. Studer und E. Böhni, Experimentelle Erfahrungen mit dem Antituberculoticum „Rimifon” 226

Fust, B., Diskussion über die Rimifon-Vorträge 295

Gloor, H. U., Urogenitaltuberkulose 419

Grob, W., Diskussion über die Rimifon-Vorträge 293

Grogg, E., Die experimentelle Rattentuberkulose unter Einfluß von Vitamin D2 98

Güntert, W., Funktionelle Röntgendiagnostik mit einem neuartigen Universal|Schirmbild-Seriengerät 213

Haefliger, E., Die Bedeutung der hämatogenen Streuung für die Entwicklung der Lungentuberkulose 446

Heî́n, J. und K.-G. Stepf, Zum Problem des sogenannten neurovegetativen Lungentonus 1

Held, E., Die gynäkologische Tuberkulose 411 Hírsch, A. und J. M. Kapferer, Ungewöhnliche Erweiterung der Trachea als Tuberkulosefolge 136 Hurní, H., Zum Wirkungsmechanismus der Thiosemicarbazone 
Jaccard, G., vide Löffler, W.

Jaquier, P., vide Delachaux, A.

Kapferer, J. M., vide Hírsch, A.

Kípfer, R. und J. Schmíd, Beitrag zur Frage der Inkubationszeit und ihrer

dissoziierten Phasen bei der Lungentuberkulose

65

Kohler, E., Quelques difficultés à propos du diagnostic et du traitement des

tuberculoses sacro-iliaques 326

Löffler, W. und G. Jaccard, Zur Klinik der hämatogenen tuberkulösen

Streuphasen 482

Morín, J., Premieres observations de malades traités par un nouvel agent

chimique 243

Morín, J., Discussion 552

Naef, A. P., Quelques remarques à propos de I'exérèse pour tuberculose

pulmonaire $\quad 540$

Nicodemí, E., Osservazioni di spirometria delle caverne tubercolari del

polmone e rapporti tra i valori spirometrici ed il quadro stratigrafico e

cavernografico

29

Ott, A., Epidemiologische und volkswirtschaftliche Probleme und Aufgaben

der Tuberkulosebekämpfung Supplementum

Pulver, R., vide Reglí, J.

Pulver, R. und G. Wilhelmí, Über den Einf luß von Pyrazolen, insbesondere von Irgapyrin, auf die Blutkonzentration der p-Aminosalicylsäure (Retard-

wirkung)

86

Ramer, Z., vide Tanner, E.

Reglí, J. und R. Pulver, Über Irgapyrin als Retardmittel 312

Reynier, J. P. de, A propos d'un cas de mastoïdite tuberculeuse chez un enfant de 14 mois 336

Sanz, M. C, vide Wílhelmí, E.

Schmid, E., Diskussion über die Rimifon-Vorträge 292

Schmíd, J., vide Kípfer, R.

Schoch, A., Vorläufige Erfahrungen mit Rimifon bei Haut- und Genitaltuberkulose 281

Schwarz, H., Weitere pathologisch-anatomische Untersuchungen über die mit

Streptomycin und PAS behandelte Meningitis tuberculosa 167

Sery, Z., vide Vojtek, V.

Summer, E., Diskussion 555

Steiner, P. M., Des indications de I'exérèse pulmonaire dans la tuberculose 532 Steínlin, H. und

E. Wílhelmí, Die Wirkung von p-(di-n-propylsulfamyl)-Ben-

zoesäure (Benemid) auf den PAS-Serum-Spiegel und seine Verwendbar-

keit in der Therapie der Tuberkulose 306

Stepf, K.G., vide Heín, J. Studer, A., vide Fust, B.

Sturzenegger, H., Diskussion 553

Tanner, E., Komplikationen nach Lungenresektion 536

Tanner, E., J. Wanner, H. Wehrlín und Z. Ramer, Rimifon in der Behandlung

der Tuberkulose; erste klinische Beobachtungen 255

Trendelenburg, F., vide Düggelí, 0 . 
Uehlinger, E., Pathologische Anatomic und Pathogenese der hämatogenen Tuberkulose 455

Vojtek, V. und Z, Sery, Die extrapleurale Pneumolyse im Kindesalter

Wanner, J., vide Tanner, E.

Wedekind, Th. und E. Wetzels, Eine neue Methode zur Herstellung von

Röntgenschichtaufnahmen 129

Wehrlin, H., Zur Diagnose und konservativen Therapie der Knochen- und

Gelenkstuberkulose 387

Wehrlín, H., vide Tanner, E.

Wernlí-Haessíg, A., Einführung in den 4. ärztlichen Fortbildungskurs der

Zürcher Kantonalen Liga gegen die Tuberkulose $\quad 347$

Wetzels, E, vide Wedekind, Th.

Widmer, H.-K., Über die Prognose der offenen Lungentuberkulose bei Kin-

dern und Jugendlichen 147

Widmer, H.-U., Uber die Kerato-Conjunctivitis skrofulosa beim Kinde und ihre Bedeutung für die tuberkulose Allgemeinerkrankung

Wílhelmí, G., vide Pulver, R.

Wílhelmí, E. und M. C. Sanz, Uber die Verzögerung der PAS-Ausscheidung durch p-(di-n-propylsulfamyl)-Benzoesäure (Benemid) 296

Wílhelmí, E., vide Steínlin, H.

Wíßler, H., Die Halsdrüsen-Tuberkulose $\quad 350$

Referate - Analyses - Abstracts

Kípfer, K., Sammelreferat über die skandinavische Tuberkulose-Literatur 1949/1950 193, 556

Wehrlín, H., Sammelreferat der deutschen Tuberkuloseliteratur 1950. . . 60, 115

Gesellschaftsberichte - Sociétés - Society Transactions

Schweizerische Vereinigung gegen die Tuberkulose, Gesellschaft Schweizerischer Tuberkuloseärzte: 32. wissenschaftliche Tagung in Lausanne, 26. und 27. April 1952. Association Suisse centre la Tuberculose, Société des Médecins Suisses specialises en Tuberculose: 32e reunion scientifique à Lausanne, les 26 et 27 avril $1952 \quad 505,201$

4. ärztlicher Fortbildungskurs der Zürcher Kantonalen Liga gegen die Tuber kulose in Arosa vom 6. bis 8. März 1952345

Libri 70, 125, 200, 343, 499

Varia 72,128

Alle Rechte vorbehalten Tous droits reserves

Printed in Switzerland

Buchdruckerei Gasse $\Gamma$ \& Cie. AC, Basel

Cliches: Sterner \& Cie., Basel 